\title{
Microfranquicia en energías renovables: Factores de éxito de la rentabilidad de los microfranquiciados - Un abordaje de regresión múltiple
}

\author{
Renewable Energy Microfranchise: Microfranchisee Profitability Success Factors - A \\ Multiple Regression Approach
}

$\begin{array}{lr}\text { Artículo recibido en diciembre } 2020 & \text { nsierrau@ @mail.com } \\ \text { Arbitrado en febrero } 2021 & \text { ORCID: 0000-0002-8059-3402 } \\ \text { Aceptado en febrero } 2021 & \text { Université Catholique de Louvain } \\ \text { Publicado en mayo } 2021 & \text { Louvain-la-Neuve, Bélgica }\end{array}$

RESUMEN

Palabras clave:

La Microfranquicia "Más Energía" es un negocio inclusivo que ha dotado de energía a más 10.000 familias bolivianas, se sabe que la rentabilidad que logren sus microfranquiciados es importante para su sostenibilidad. El objetivo de esta investigación fue analizar los factores explicativos de su rentabilidad y su importancia comparativa, a través de un análisis descriptivo y de regresión lineal múltiple de mínimos cuadrados aplicado al $98 \%$ del total de microfranquiciados esparcidos en 6 departamentos de Bolivia, en el período 2016-2017.

Los resultados muestran que los microfranquiciados incrementaron su rentabilidad en $79 \%$, asimismo las variables que explican el $72 \%$ de su rentabilidad son: prioridad de la población a la energía domiciliaria, competencia, publicidad, su reputación, edad y días trabajados al mes. Los hallazgos encontrados significan una importante contribución a la discusión porque aportan con un enfoque cuantitativo explicativo y de priorización de variable, que no se encontró en la bibliografía.

Microfranquicia; Rentabilidad de microfranquiciados; Negocios inclusivos; Modelos de desarrollo sostenible; Energía renovable; Regresión múltiple

\footnotetext{
ABSTRACT

"Más Energía" Microfranchise is an inclusive business that has provided energy to more than 10,000 Bolivian families. It is known that the profitability achieved by its microfranchisees is important for its sustainability. For this reason, the research proposed to analyze the explanatory factors of its profitability and what was its comparative importance, through a descriptive analysis and the multiple linear regression of least squares applied to $98 \%$ of the total of microfranchisees scattered in 6 departments of Bolivia, in the period 2016-2017.

The results show that microfranchisees increased their profitability by $79 \%$, likewise the variables that explain $72 \%$ of their profitability are: priority of the population to home energy, competition, advertising, their reputation, age, and days worked per month.

The findings found represent an important contribution to the discussion because they provide a quantitative explanatory and variable prioritization approach, which was not found in the bibliography.

Keywords: $\quad$ Profitability of microfranchisees; Inclusive Businesses; Sustainable development models; Renewable energies; Multiple Regression
} 


\section{INTRODUCCIÓN}

Energética es una Organización No Gubernalmental de Bolivia, con más de 20 años de experiencia en energías renovables. En septiembre de 2013, con el apoyo del Fondo Multilateral de Inversiones (FOMIN) del Banco Interamericano de desarrollo (BID), se puso en marcha un negocio inclusivo desarrollado por esta empresa: la Microfranquicia (MC) "Más Energía" (+e), que busca facilitar el acceso a sistemas de energía renovables a familias de las zonas rurales de Bolivia que no tienen cobertura eléctrica, en los departamentos de Oruro, Potosí, Cochabamba, Chuquisaca, Santa Cruz y La Paz.

La MC es comercial y de servicios, cuenta con el microfranquiciador $+\mathbf{e}$ y una red de microfranquiciados (mcs). te se ocupa de la provisión de equipos y accesorios, el desarrollo de los sistemas de gestión y comercialización y el desarrollo de la red de mcs; éstos últimos se encargan de realizar las ventas y servicios en los municipios donde trabaja $+\mathbf{e}$, los mcs para mejorar su trabajo cuentan con el apoyo de un grupo de asesores.

Los principales productos que oferta $+e$ son picolámparas y sistemas solares de litio para hogares.

La MC tiene dos procedimientos de venta: la Venta por Distribución (ventas por convenios con los Gobiernos Autónomos Municipales (GAM): $25 \%$ aporte de GAM, 25\% de Energética y 50\% del cliente); y la Venta Directa (ventas y servicios realizados directamente por los mcs). A diciembre de 2017, las Ventas por Distribución representaban el $86 \%$ del negocio, y las Ventas Directas el $14 \%$ restante; +e ha dotado de energía a más de 10.000 familias y ha constituido una MC con una red de 60 mcs apoyados por 5 asesores.
Se sabe que +e es rentable, aunque con fuertes dificultades financieras, sin embargo, no se conoce a profundidad sobre la rentabilidad de los mcs (Sierraalta et al., 2017). Por tanto, el objetivo de este estudio fue determinar cuáles han sido los factores explicativos de la rentabilidad de los microfranquiciados y cuál ha sido la importancia comparativa de estos factores.

La investigación se justifica y cobra relevancia porque para la continuidad y sostenibilidad del emprendimiento es "indispensable" que los mcs logren rentabilidad; caso contrario, éstos dejarían de trabajar en la MC y ésta tendría dificultades para continuar como tal. Asimismo, es importante porque +e para continuar, optimizar y crecer en su red de mcs, debe entender cuáles son las variables críticas que explican la rentabilidad de los mcs y cuál es la importancia comparativa de estos.

Entonces, existe una aplicación práctica inmediata, ya que la investigación se utilizará para la toma de decisiones respecto de la selección de mcs y las variables críticas para lograr su rentabilidad.

Por otra parte, se convierte en un aporte teórico para el estudio de las MCs en todo el mundo, dado que la investigación ha aportado al análisis de las MCs con un enfoque cuantitativo, explicativo y de priorización de variables de la rentabilidad de los mcs. Nótese que da cuenta de una característica muy específica, el modelo de negocio de $+\mathbf{e}$ no es habitual en otros negocios de MCs.

En la bibliografía revisada se ha constatado que lograr y analizar la rentabilidad de los mcs dentro de un modelo de negocio de MC es muy importante. Lehr (2008), afirma que la rentabilidad de los mcs es vital para la 
permanencia del negocio, Magleby (2017), indica que mcs exitosos podrán operar autosuficientemente gracias a su rentabilidad, y Beck et al. (2010), resaltan que la ausencia de rentabilidad para para ellos pone en riesgo la MC, Hurlimann (2011), va más allá y afirma que es necesario que el margen de utilidad para el $\mathrm{mc}$ sea lo suficientemente grande para mantenerlo motivado $y$, en este mismo sentido, Burand y Koch (2010) remarcan la importancia de la rentabilidad para los mcs. Asimismo, existen metodologías que explican cómo se debería contabilizar la rentabilidad del mc (Conde, 2013).

Lehr (2008), encuentra que son tres las variables que serían fundamentales para su éxito: financiamiento, rapidez en el logro de rentabilidad y fuentes de ingresos adicionales para lograr sostenibilidad. Por su parte Hurlimann (2011), ha identificado las siguientes variables para explicar el éxito de los mcs: a) el trabajo cara a cara del mc con el cliente; b) demanda suficiente de baja densidad poblacional y bajo ingreso; c) demanda periódica; d) problema de acceso importante; e) precios adecuados de los productos y servicios; f) financiamiento; g) agregación de negocios; h) ingresos suficientes; i) ingresos cuasi-continuos; j) ingresos de rápido crecimiento $y_{;}$k) personalidad y el respeto por el $\mathrm{mc}$.

\section{METODOLOGÍA}

Se trata de una investigación cuantitativa con alcance explicativo, que generó un modelo que define las variables que explican la rentabilidad, y las prioriza según su importancia. Los sujetos de la investigación fueron 48 mcs activos en el período de 05/2016 a 05/2017, en ese momento +e tenía dos años de evolución (que se consideraba un periodo de vida razonable para una evaluación). Los 48 sujetos constituyeron el $98 \%$ de la totalidad de mcs, en ese sentido no se trabajó con una muestra, sino, prácticamente, el total de la población estudiada.

Se consideró como referente la relación mínima número de observaciones por número variables de 5:1, cumpliendo la regla general de Hair (Hair et al., 2014).

\section{Variables y técnicas para la descripción de la suficiencia de rentabilidad}

Todas las variables de la investigación se definieron en análisis con los asesores de $+\mathbf{e}$. Dado que se buscó describir el comportamiento de la suficiencia de rentabilidad se utilizó técnicas descriptivas. Las variables que se utilizaron para analizar la suficiencia de la rentabilidad fueron: Incremento Utilidad Familiar en \%/año (IUF), Utilidad del mc en Bs/año (Umc) y Utilidad Familiar en Bs/año (UF). Todos los datos fueron tomados de los reportes de ventas mensuales de mcs.

\section{Variables y técnicas para la explicación de la rentabilidad}

Para analizar las variables que explican la rentabilidad se utilizó como la técnica de regresión lineal múltiple de mínimos cuadrados ordinarios (MCO). Se analizó la explicación de la rentabilidad a través de tres regresiones, que en términos de modelo teórico expresan las siguientes hipótesis:

\section{Regresión de la Utilidad del mc}

Umci $=\beta 1+\beta 2$ VSEci $+\beta 3$ PNBSci $+\beta 4$ PGAMci $+\beta 5 \mathrm{PUi}+\beta 6 \mathrm{dFi}+\beta 7 \mathrm{dCi}+\beta 8 \mathrm{dMi}+$ $\beta 9 \mathrm{Ai}+\beta 10 \mathrm{Ei}+\beta 11 \mathrm{Ti}+\beta 12 \mathrm{Ri}+\beta 13 \mathrm{HAi}+\beta 14$ $\mathrm{PEPi}+\beta 15 \mathrm{CO}+\beta 16 \mathrm{ESi}+$ 
Regresión del Ingreso Bruto por Venta

YBVi $=\beta 1+\beta 2$ VSEci $+\beta 3$ PNBSci $+\beta 4$

PGAMci $+\beta 5 \mathrm{PUi}+\beta 6 \mathrm{dFi}+\beta 7 \mathrm{dCi}+\beta 8 \mathrm{dMi}+$

$\beta 9 \mathrm{Ai}+\beta 10 \mathrm{Ei}+\beta 11 \mathrm{Ti}+\beta 12 \mathrm{Ri}+\beta 13 \mathrm{HAi}+\beta 14$

$\mathrm{PEPi}+\beta 15 \mathrm{CO}+\beta 16 \mathrm{ESi}+\varepsilon \mathrm{i}$

Regresión del Ingreso Bruto por

Distribución

YBDi $=\beta 1+\beta 2$ PEAi $+\beta 3$ PEPi $+\beta 4$

PGAMci $+\beta 5$ VSEci $+\beta 6$ PNBSci $+\beta 7 \mathrm{dCDi}+$

$\beta 8 \mathrm{Ri}+\beta 9 \mathrm{PUi}+\varepsilon \mathrm{i}$
Se generó con las bases de datos de Energética, del Estado Plurinacional de Bolivia y encuestas a los mcs. Las variables que se tomaron en cuenta se operativizan en la tabla 1.

\section{Tabla 1}

Variables para analizar las regresiones

\begin{tabular}{|c|c|c|c|c|}
\hline Nombre de la Variable & Sigla & $\begin{array}{l}\text { Tipo de } \\
\text { variable }\end{array}$ & $\begin{array}{l}\text { Unidad de } \\
\text { medida }\end{array}$ & $\begin{array}{l}\text { Hipótesis } \\
\text { de relación }\end{array}$ \\
\hline Ingreso Bruto Ventas & YBV & $\begin{array}{l}\text { Dependiente } \\
\text { Determinística, } \\
\text { Métrica }\end{array}$ & Bs/año & \\
\hline Ingreso Bruto Distribución & YBD & $\begin{array}{l}\text { Dependiente } \\
\text { Determinística, } \\
\text { Métrica }\end{array}$ & Bs/año & Directa \\
\hline Publicidad en radioemisoras & PU & $\begin{array}{l}\text { Independiente } \\
\text { Métrica }\end{array}$ & Pases/año & Directa \\
\hline Viviendas sin Energía Eléctrica & VSEC & $\begin{array}{l}\text { Independiente } \\
\text { Métrica }\end{array}$ & Cien viviendas & Directa \\
\hline $\begin{array}{l}\text { Presupuesto Gobierno } \\
\text { Autónomo Municipal }\end{array}$ & PGAMc & $\begin{array}{l}\text { Independiente } \\
\text { Métrica }\end{array}$ & Cientos Bs/año & Directa \\
\hline $\begin{array}{l}\text { Población con Necesidades } \\
\text { Básicas Satisfechas }\end{array}$ & PNBSC & $\begin{array}{l}\text { Independiente } \\
\text { Métrica }\end{array}$ & Cien personas & Directa \\
\hline Días feria & $\mathrm{dF}$ & $\begin{array}{l}\text { Independiente } \\
\text { Métrica }\end{array}$ & Días/mes & Directa \\
\hline Días comunidad & $d C$ & $\begin{array}{l}\text { Independiente } \\
\text { Métrica }\end{array}$ & Días/mes & Directa \\
\hline Días mes & $\mathrm{dM}$ & $\begin{array}{l}\text { Independiente } \\
\text { Métrica }\end{array}$ & Días/mes & Directa \\
\hline
\end{tabular}




\section{Tabla 1}

Variables para analizar las regresiones

\begin{tabular}{|c|c|c|c|c|}
\hline Nombre de la Variable & Sigla & $\begin{array}{l}\text { Tipo de } \\
\text { variable }\end{array}$ & $\begin{array}{l}\text { Unidad de } \\
\text { medida }\end{array}$ & $\begin{array}{l}\text { Hipótesis } \\
\text { de relación }\end{array}$ \\
\hline Antigüedad & $A$ & $\begin{array}{l}\text { Independiente } \\
\text { Métrica }\end{array}$ & Meses & Directa \\
\hline Edad & $\mathrm{E}$ & $\begin{array}{l}\text { Independiente } \\
\text { Métrica }\end{array}$ & Años & Inversa \\
\hline Tienda & $\mathrm{T}$ & $\begin{array}{l}\text { Independiente } \\
\text { NO métrica }\end{array}$ & $\begin{array}{l}0=\mathrm{No} \\
1=\mathrm{Si}\end{array}$ & Directa \\
\hline Reputación & $\mathrm{R}$ & $\begin{array}{l}\text { Independiente } \\
\text { NO métrica }\end{array}$ & $\begin{array}{l}1=\text { Poca } \\
2=\text { Aceptable } \\
3=\text { Buena }\end{array}$ & Directa \\
\hline Habilidades & $\mathrm{HA}$ & $\begin{array}{l}\text { Independiente } \\
\text { NO métrica }\end{array}$ & $\begin{array}{l}1=\text { Poca } \\
2=\text { Regular } \\
3=\text { Buena } \\
4=\text { Muy buena }\end{array}$ & Directa \\
\hline Prioridad de población a Energía & PEP & $\begin{array}{l}\text { Independiente } \\
\text { NO métrica }\end{array}$ & $\begin{array}{l}1=\text { Baja } \\
2=\text { Media } \\
3=\text { Alta }\end{array}$ & Directa \\
\hline Competencia & $\mathrm{CO}$ & $\begin{array}{l}\text { Independiente } \\
\text { NO métrica }\end{array}$ & $\begin{array}{l}1=\text { Poca } \\
2=\text { Regular } \\
3=\text { Mucha }\end{array}$ & Inversa \\
\hline Estudios & ES & $\begin{array}{l}\text { Independiente } \\
\text { NO métrica }\end{array}$ & $\begin{array}{l}1=\text { Ninguno } \\
2=\text { Escuela } \\
3=\text { Colegio } \\
4=\text { Mas que } \\
\text { colegio }\end{array}$ & Directa \\
\hline Días comunidad asesor y mc & $d C D$ & $\begin{array}{l}\text { Independiente } \\
\text { Métrica }\end{array}$ & Días/mes & Directa \\
\hline $\begin{array}{l}\text { Prioridad de Autoridades } \\
\text { Municipales a Energía }\end{array}$ & PEA & $\begin{array}{l}\text { Independiente } \\
\text { NO métrica }\end{array}$ & $\begin{array}{l}1=\text { Baja } \\
2=\text { Media } \\
3=\text { Alta }\end{array}$ & Directa \\
\hline
\end{tabular}


Se aclara que en la Regresión de Utilidad del $\mathrm{mc}$, la variable Ingreso Bruto por Ventas no fue incluida como variable independiente porque la relación con la variable dependiente es funcional o determinística.

\section{Generación de resultados}

Una vez realizada la corrección de errores en los datos se aplicó la técnica de selección Backward elimination con un nivel de significancia del 0,05 para obtener una regresión inicial, que luego fue controlada y corregida de outliers (gráficos, residuos estandarizados, distancia de Cook, distancia de ajuste); de apalancadores (técnica de apalancamiento); de normalidad (gráficos, test de Shapiro-Wilk); de homocedasticidad (gráficas, test de White, test de Breusch-Pagan); de multicolinealidad (factor de inflación de varianza); de linealidad (gráficos, selección de transformaciones adecuadas); y de comprobación de validez del modelo. Después de este proceso se obtuvo una regresión final controlada y corregida que cumple con los supuestos de MCO. La regresión final fue comparada con Regresiones con Errores Estándar Robustos, Robusta y Cuartílica, buscando resultados alternativos mejores.

Para encontrar la regresión del Ingreso Bruto por Distribución se aplicaron tres métodos de selección automática Forward Selection, Backward elimination y Stepwise regression, sin resultados satisfactorios. Por esta razón; considerando, de mayor a menor, la correlación entre la variable dependiente y las variables independientes, se generaron varios modelos, siendo el que se presenta en los resultados el más satisfactorio.

Los resultados fueron discutidos en reuniones colectivas e individuales con los asesores de $+\mathbf{e}$, para realizar correcciones y arribar a conclusiones.

\section{RESULTADOS}

\section{Rentabilidad de los mcs}

Los mcs han incrementado anualmente su utilidad familiar promedio en $79 \%$, más que el $20 \%$ planteado como meta por el convenio BID - Energética. Sin embargo, este promedio no representa adecuadamente el comportamiento de los mcs, porque tiene a una alta variabilidad (coeficiente de variación de 172\%). Así, los percentiles son indicadores más adecuados; considerándolos se concluye que la mitad de los mcs han incrementado su utilidad en más del $20 \%$, además el cuartil superior muestra que los mcs han aumentado su utilidad desde el $105 \%$, hasta el $603 \%$.

\section{Ingresos de los mcs}

Se presentan los ingresos de los mcs de $+\mathbf{e}$, para, posteriormente, compararlos con datos de otros encontrados en la bibliografía. El Salario Mínimo Nacional Diario (SMNd), en Bolivia el 2016 fue de Bs 82/día y se considera un referente de comparación adecuado ${ }^{13}$. Tomando, los percentiles, se concluye que la mitad de los mcs han alcanzado 33 veces más el SMNd; además el $25 \%$ de los mcs más destacados lograron desde 84 hasta 749 veces el SMNd.

\section{Factores explicativos de la rentabilidad de los mcs}

El modelo de regresión múltiple por $\mathrm{MCO}$

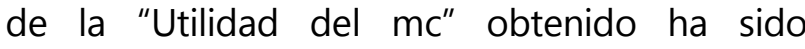
estadísticamente significativo $(F=10,82$, Prob $F=$ 0.0000, Nivel de significación $=0,005)$; $y$ ha explicado, aproximadamente, el $72 \%$ de la 
variabilidad de la Utilidad. Tomando en cuenta el número de predictores incorporados, 8 en el modelo, éste explica el $66 \%$ de la variabilidad de la Utilidad. Este modelo se ha elaborado con 43 observaciones y 33 grados de libertad.

Como se muestra en la tabla 2, el modelo cumplió con los supuestos de los MCO: parte del resultado de un proceso stepwise bacward, retiró 5 outliers, luego se transformó en semilogarítmico (para cumplir con la normalidad), fue homocedástico y por razones de multicolinealidad se retiró una variable. Asimismo, los ocho coeficientes del modelo fueron estadísticamente significativos porque tienen $p$ - values menores al nivel de significación, de 0,05. De los ocho coeficientes resultantes, dos tienen resultados ilógicos y están en cursiva (la hipótesis del modelo era que estas variables independientes tenían una relación directa con la variable dependiente, pero los coeficientes del resultado al ser negativos indicaron relaciones inversas).

En el lado derecho de la tabla 2 se presenta la priorización de las variables independientes, considerando su contribución a la variabilidad de la Utilidad, en términos de desviación estándar. La variable más importante fue la prioridad alta que dieron los pobladores de los municipios, a la energía eléctrica. Al final de la tabla 2 se presentan modelos alternativos $\mathrm{y}$ las razones por las que fueron rechazados comparativamente.

\section{Tabla 2}

Utilidad - Parámetros estimados y orden de importancia

\begin{tabular}{lcclcc}
\hline \multicolumn{1}{c}{ Modelo regresión semilogarítmico } & \multicolumn{3}{c}{ Importancia de las variables (en desviaciones } \\
estándar)
\end{tabular}

Según se ve en la tabla 2, las variables referidas a determinar si posee una tienda y si tiene estudios superiores, dan como resultado relación inversa, por tanto, son consideradas ilógicas, el análisis a profundidad se realiza en el apartado de discusión. También se debe notar que, en el caso de las variables independientes, para las transformaciones de las variables continuas se usa las recomendaciones de Jacoby (2017). 


\section{Control y ajuste por supuestos}

- Outliers: Retiro de 5 observaciones.

- Normalidad: Cambio de variable dependiente de "Utilidad" por "logaritmo de Utilidad" Homocedasticidad: El modelo es homocedástico.

- Multicolinealidad: Se retira la variable "Presupuesto Gobierno Autónomo Municipal"

- Linealidad: Las relaciones entre variable dependiente e independientes es lineal.

Se presentan modelos alternativos y las razones por las que fueron rechazados comparativamente: 1) Regresión con Errores
Estándar Robustos: No mejora el modelo, 2) Regresión Robusta: Modelo con más variables significativas, pero con más variables ilógicas Regresión y, 3) Cuartílica: Modelo con más variables significativas, pero con más variables ilógicas.

Los asesores de $+\mathbf{e}$ consideraron que, para una mejor comprensión y discusión de los resultados del modelo semilogarítmico, se transformen las variables independientes a moneda nacional (bolivianos) y se ordenen en función de cuanto impactan, en bolivianos, en la utilidad.

En la tabla 3 se analiza la importancia de las variables, valorando por orden de importancia los parámetros estimados.

\section{Tabla 3}

Utilidad - Parámetros estimados transformados (en bolivianos) y orden de importancia

\begin{tabular}{lcc}
\hline Importancia de las variables (en bolivianos) & & \\
\hline \multicolumn{1}{l}{ Variables } & $\begin{array}{c}\text { Coeficiente } \\
\text { (Bs) }\end{array}$ & Importancia \\
Dependiente & & \\
Utilidad mc & & \\
Independientes & 276 & $1^{\circ}$ \\
Prioridad de Población a Energía: Alta & 208 & $2^{\circ}$ \\
Competencia: Regular & 129 & $3^{\circ}$ \\
Reputación: Buena & 77 & $4^{\circ}$ \\
Días al mes & 69 & $5^{\circ}$ \\
Edad & 67 & $6^{\circ}$ \\
Publicidad & -24 & $7^{\circ}$ \\
Tienda & -9 & $8^{\circ}$ \\
Estudios más que colegio & & \\
\hline
\end{tabular}

Los resultados de la tabla 3 indican que, siendo constantes las otras variables, con una certeza mayor al $95 \%$, se puede indicar que los mcs: 1) en municipio donde los pobladores dieron alta prioridad a la energía eléctrica, tuvieron una utilidad anual mayor en Bs 276 que en los municipios donde los pobladores dieron una baja prioridad; 2) en municipios donde hubo una regular competencia, se tuvo utilidad anual mayor en Bs 208, que en los que 
hubo poca competencia; 3) en municipios donde los mcs gozaron de buena reputación, se tuvo utilidad anual mayor en Bs 129, que en los que tuvieron poca reputación; 4) los que trabajaron un día más al mes en la $M C$, incrementaron su utilidad anual en Bs 77; 5) con un año más de edad incrementaron su utilidad en Bs 69; 6) los que pusieron un pase de publicidad más en el año, incrementaron su utilidad en Bs 67, siendo constantes las otras variables; 7) los que sí tenían tienda, han tenido una utilidad anual menor en Bs 24 que los que no tenían tienda; 8) los que han acabado su educación en el colegio, han tenido una utilidad anual menor en Bs 9 que los que no han tenido ninguna educación.

\section{Ingreso Bruto por Ventas}

Se puede observar la relación de variables del modelo ajustado del Ingreso bruto por ventas, según tabla 4.

\section{Tabla 4}

Ingreso Bruto por Venta Directa - Ajuste general del modelo

$\begin{array}{lr}\text { Número de observaciones } & 43 \\ \text { F(5,37) } & 14,13 \\ \text { Grados de libertad } & 37 \\ \text { Nivel de significación } & 0,05 \\ \text { Prob > F } & 0,0000 \\ \text { R2 } & 0,6664 \\ \text { Adj R2 } & 0,6099\end{array}$

Según se muestra en la tabla 4, el modelo de regresión múltiple por MCO del "Ingreso Bruto por Venta Directa" obtenido fue estadísticamente significativo $(F=14,13$, Prob $F=$ $0,0000$, Nivel de significación $=0,005)$; y explicó aproximadamente el $67 \%$ de la variabilidad del Ingreso Bruto por Venta Directa. Ajustando la variabilidad por el número de predictores incorporadas, 5 en el modelo, éste explica el $61 \%$ de la variabilidad del Ingreso Bruto por Ventas. Este modelo se ha elaborado con 43 observaciones y 37 grados de libertad.

Como se muestra en la tabla 5 , el modelo que ha cumplido con los supuestos de los MCO parte del resultado de un proceso stepwise bacward que ha retirado 5 observaciones (por ser outliers y apalancadores); cumple con los supuestos de normalidad, homocedasticidad y multicolinealidad; sin embargo, necesita una corrección de la variable "días al mes" por su raíz cuadrada para cumplir con el supuesto de linealidad. Los cinco coeficientes del modelo son estadísticamente significativos porque tienen $\mathrm{p}$ values menores al nivel de significación $(0,05)$. De los cinco coeficientes resultantes, uno ha tenido resultado ilógico y está en cursiva (la hipótesis del modelo era que esta variable independiente tenía una relación directa con la variable dependiente, pero el coeficiente del resultado al ser negativo ha indicado una relación inversa).

Considerando su contribución a la variabilidad de la Utilidad (en términos de desviación) se presentan las variables 
independientes por orden de importancia, este orden coincide con la importancia práctica de las variables que dan los asesores de $+\mathbf{e}$ en función del impacto en bolivianos al Ingreso Bruto por Venta Directa.

Los resultados de la tabla indican que, siendo constantes las otras variables, con una certeza mayor al 95\%, se puede indicar sobre los mcs: 1) los que tuvieron buenas habilidades, realizaron ventas directas mayores en Bs 4.225 que los que tuvieron habilidades regulares: 2 ) los que trabajaron un día más al mes en las comunidades, tuvieron ventas directas mayores en $\mathrm{Bs} 4.018$; 3) los que trabajaron un día más al mes en las ferias, tuvieron ventas directas mayores en Bs 1.518; 4) en los municipios en los que los pobladores dieron una media prioridad a la energía eléctrica, tuvieron ventas directas menores en Bs 4.184 que en los municipios donde los pobladores dieron una baja prioridad; 5) los que gozaron de aceptable reputación, tuvieron ventas directas menores en Bs 3.014 que los que tuvieron poca reputación.

\section{Tabla 5}

Ingreso Bruto Venta Directa - Parámetros estimados y orden de importancia

\begin{tabular}{|c|c|c|c|c|c|c|}
\hline \multicolumn{4}{|c|}{ Modelo de regresión } & \multicolumn{3}{|c|}{$\begin{array}{l}\text { Importancia de las variables } \\
\text { (en desviaciones estándar) }\end{array}$} \\
\hline Variables & $\begin{array}{l}\text { Coef } \\
\text { (Bs) }\end{array}$ & $p>|t|$ & $\begin{array}{l}\text { Importancia } \\
\text { (Bs) }\end{array}$ & Zvariable & Coef & Importancia \\
\hline Dependiente & & & & Dependiente & & \\
\hline Ingreso Bruto Ventas & & & & zIngreso Bruto Ventas & & \\
\hline Independientes & & & & Independientes & & \\
\hline Habilidades Buenas & 4.426 & 0,007 & $1^{\circ}$ & Habilidades Buenas & 0,60 & $1^{\circ}$ \\
\hline Días comunidad & 4.018 & 0,001 & $2^{\circ}$ & zDías comunidad & 0,40 & $2^{\circ}$ \\
\hline Días feria & 1.519 & 0,008 & $3^{\circ}$ & zDías feria & 0,28 & $3^{\circ}$ \\
\hline $\begin{array}{l}\text { Prioridad Población a Energía: } \\
\text { Media }\end{array}$ & -4.184 & 0,013 & $4^{\circ}$ & $\begin{array}{l}\text { Prioridad Población a } \\
\text { Energía: Media }\end{array}$ & $-0,57$ & $4^{\circ}$ \\
\hline Reputación Aceptable & -3.015 & 0,048 & $5^{\circ}$ & Reputación Aceptable & $-0,41$ & $5^{\circ}$ \\
\hline
\end{tabular}

Según se observa en la tabla 5 , es importante recalcar que las variables prioridad de la población a la energía y reputación fueron consideradas variables ilógicas, debido a que representan relación inversa (serán analizadas en el apartado de discusión). Destáquese también que la regresión cuartílica ha resultado en un modelo interesante de analizar. Se recalca que para las transformaciones de las variables continuas se usa las recomendaciones de Jacoby (2017).

\section{Control y ajuste por supuestos}

- Outliers: Retiro de 4 observaciones Apalancadores: Retiro de 1 observación Normalidad: El modelo es normal.

- Homocedasticidad: El modelo es homocedástico. 
- Multicolinealidad: El modelo no tiene multicolinealidad.

- Linealidad: La relación entre "Ingreso Bruto Ventas" y "Días al mes" no es lineal. Esta última se transforma por su raíz cuadrada.

Se presentan modelos alternativos y las razones por las que fueron rechazados comparativamente: 1) Regresión con Errores
Estándar Robustos: no mejora el modelo Regresión Robusta, no tiene más variables significativas; 2) Regresión Cuartílica: puede ser un modelo alternativo interesante de analizar.

\section{Ingreso Bruto por Distribución}

El ajuste general del modelo de ingreso bruto por distribución se presenta en la tabla 6 .

\section{Tabla 6}

Ingreso Bruto por Distribución - Ajuste general del modelo

$\begin{array}{lr}\text { Número de observaciones } & 10 \\ \text { F(1,8) } & 93,96 \\ \text { Grados de libertad } & 8 \\ \text { Nivel de significación } & 0,05 \\ \text { Prob > F } & 0,0000 \\ \text { R2 } & 0,9215 \\ \text { Adj R2 } & 0,9117\end{array}$

Como se puede observar en la tabla 6, el modelo de regresión múltiple por $\mathrm{MCO}$ del "Ingreso Bruto por Distribución" obtenido ha sido estadísticamente significativo ( $F=93,96$, Prob $F=0,0000$, Nivel de significación $=0,005)$; y ha explicado, aproximadamente, el $92 \%$ de la variabilidad del Ingreso Bruto por Distribución. Ajustando la variabilidad por el número de predictores incorporados, 1 en el modelo, éste explica el 91\% de la variabilidad del Ingreso por Distribución. Este modelo se ha logrado con 10 observaciones y 8 grados de libertad.

Asimismo, se presenta en la tabla 7 el modelo que cumplió con todos los supuestos de los MCO, luego de haber retirado 2 observaciones, por ser outliers; es decir, es normal, homocedástico, no tiene multicolinealidad y es lineal.

\section{Tabla 7}

Ingreso Bruto por Distribución - Parámetro estimado

\section{Modelo de regresión}

\section{Variables}

Coeficiente $p>|t|$

\section{Dependiente}

Ingreso Bruto Distribución

\section{Independientes}

Prioridad de Autoridades Municipales a Energía: Alta

$509.136,000$ 
El único coeficiente del modelo; es estadísticamente significativos ( $p$-value = $0,0000)$ y lógico, indica que mcs, en los municipios en los que las autoridades municipales dieron una alta prioridad a la energía eléctrica, tuvieron ventas por distribución mayores en Bs 509.136,000 que en los que las autoridades dieron una baja prioridad. Los modelos alternativos, se han rechazado porque no mejoran el modelo.

\section{Control y ajuste por supuestos}

- Outliers: Retiro de 2 observaciones.

- Normalidad: El modelo es normal.

- Homocedasticidad: El modelo es homocedástico.

- Multicolinealidad: El modelo no tiene multicolinealidad.

- Linealidad: Las relaciones entre variable dependiente e independientes son lineales.

Se presentan modelos alternativos y las razones por las que fueron rechazados comparativamente: 1) Regresión con errores estándar robustos: no mejora el modelo Regresión Robusta y; 2) Regresión cuartílica: no mejora el modelo.

\section{DISCUSIÓN}

Lo indispensable de lograr la rentabilidad de los mcs para el éxito de la MC ha sido probado en las investigaciones presentadas por Lehr (2008), Magleby (2007), Beck et al. (2010), Hurlimann (2011), Fairbourne (2007), Burand y Koch (2010) y Conde (2013); la rentabilidad de los mcs de varias $\mathrm{MC}$ ha sido descrita por Lehr, 2008; asimismo, se han identificado y descrito cualitativamente las variables que explican la rentabilidad de los mcs (Lehr, 2008); (Hurlimann, 2011); (Burand y Koch) 2010; (Conde, 2013) y
(Fairbourne, 2007). Esta investigación aporta con un estudio cuantitativo, cuyos resultados se analizan a continuación.

\section{Descripción de la rentabilidad e ingresos de los mcs}

Los mcs de +e han superado en 4 veces la meta de rentabilidad del convenio BIDEnergética y que se consideraba razonable. Esta rentabilidad con márgenes suficientes (Hurlimann), perfila la sostenibilidad de +e (Lehr y Fairbourne), apuntalando una lógica ganar/ganar entre los mcs y la MC (Magleby), resultando en una disminución del riesgo de fracaso de la MC (Beck y Deeelder). A pesar de los conceptos indicados, se considera que la rentabilidad de los mcs tiene limitaciones importantes $y$ esto se refleja en una considerable tasa de deserción. La insatisfacción de los mcs, probablemente se explique porque los ingresos no son continuos, son muy espaciados en el tiempo; la administración del emprendimiento que exige la $M C$ es considerada pesada; los precios topes de venta que establece $+\mathbf{e}$ son considerados limitados $y_{\text {, }}$ en consecuencia, los márgenes de beneficio son considerados insuficientes; la competencia de los productos chinos limita las ventas; y finalmente las distancias a recorrer para la realización de las ventas, son grandes.

Comparativamente los ingresos alcanzados por mcs de +e (89 veces el salario mínimo nacional de Bolivia) han sido mejores que los logrados en Vision Spring (6 veces el salario diario de la India).

\section{Explicación de la rentabilidad de los mcs}

Se ha logrado desarrollar un modelo que explica el $72 \%$ de la utilidad de los mcs, con 5 variables lógicas, con las cuáles se puede hacer 
afirmaciones con un nivel de certeza mayor al 95\%:

- Es lógico que en los municipios en los que "la población da una prioridad alta a la energía" se realicen mayores ventas $y$, en consecuencia, "mayor utilidad". Sin embargo, como una limitación, a mejorar en otras investigaciones, se anotó que pudo existir el riesgo de que los asesores consultados confundieran la percepción de la población con la percepción de las autoridades municipales.

- La explicación a que en un municipio donde la "competencia es regular" se logre mayor "Utilidad" es la siguiente: Dado que la competencia es principalmente de productos chinos, en un primer momento, los clientes prefieren éstos porque son más baratos; luego al constatar que los productos chinos son de mala calidad prefieren los productos de $+\mathbf{e}$.

- La relación directa entre la "buena reputación" de los mcs y la "utilidad" se explica porque aquella genera confianza en la clientela.

- De igual manera al trabajar "más días" al mes, los mcs tienen más posibilidades de más ventas y en consecuencia "mayor utilidad".

- Por lógica se entiende que la probable explicación de que "a mayor edad" los mcs generen "mayor utilidad" es que, a mayor edad éstos son más responsables y están más arraigados en su región de intervención. Sin embargo, por el lado ilógico se podría indicar que, per se, los mcs "más viejos" logran "mayor rentabilidad", siendo esto un absurdo. Para mejorar la investigación se deberá limitar el rango de edad en la que esta afirmación es correcta.

- Parece lógico que "a mayor publicidad" los mcs logren "mayor utilidad", porque los productos son más conocidos, los clientes se hallan más influenciados a la compra, en consecuencia, compran más productos de $+\mathbf{e}$.

- Respecto a la importancia de las variables, se considera que la priorización lograda por el modelo es razonable, aunque con algunos matices. El orden importancia, desde una perspectiva de campo, sería: la prioridad que da la población a la energía eléctrica, la reputación de los mcs, la publicidad, la competencia, los días trabajados al mes y la edad.

Respecto de las variables identificadas en la revisión bibliográfica se indica:

- La mayoría se han incorporado en el análisis (aunque posteriormente en el modelo no aparecen porque no alcanzan el nivel de significación del 0,05). Fueron adecuadas a la especificidad de $+\mathbf{e}$, a continuación se las presenta y en paréntesis como han sido utilizadas en el modelo: Fuentes de ingreso adicionales 0 agregación de negocios (Tienda), Demanda suficiente (Viviendas sin Energía Eléctrica, Prioridad de Población a Energía, Prioridad de Autoridades Municipales a Energía), Demanda de bajos ingresos o demanda de ingresos suficientes (Población con necesidades básica satisfechas, presupuesto Gobierno Autónomo Municipal), Trabajo cara a cara (Días comunidad, días feria, días al mes, días comunidad asesor y mc), Personalidad y respeto por el $\mathrm{mc} o$ 
reputación (Reputación, edad),

Habilidades, actitudes (Habilidades,

estudios),

Precios

adecuados

(Competencia).

- Hay que destacar que la bibliográfica indica la rentabilidad, pero no se identifican las variables para medir la rentabilidad. En la investigación la variable que mide la rentabilidad es la Utilidad del microfranquiciado (Ingresos totales Gastos totales del emprendimiento de $\mathrm{MC}$ )

- El importante aporte de la investigación en comparación con la bibliografía es: tratamiento cuantitativo a las variables utilizadas, se ha establecido un orden de importancia de variables $y$ se ha identificado una relación explicativa entre rentabilidad y sus variables; superando las limitaciones identificadas en la revisión bibliográfica.

- Las variables identificadas en la bibliografía y que no se consideraron son: Demanda periódica, Demanda en rápido crecimiento, Rapidez en el logro de rentabilidad, Ingresos cuasi-continuos, Ingresos de rápido crecimiento. Inicialmente se buscó realizar un análisis con la técnica de Datos de Panel, pero se obtuvieron muchos valores perdidos resultando modelos débiles.

Asimismo, la variable Financiamiento fue considerada implícita debido a que en el período de la investigación y hasta la fecha, los mcs reciben los productos en consignación. Tampoco se consideró la variable Problema de acceso importante, por ser un supuesto básico de la MC que no era necesario incorporar.
El modelo contiene 2 variables que no son lógicas: a) En +e no parece lógico que los mcs que tienen tienda obtengan menos utilidad que los que no tienen tienda. La mayoría de los mcs que tienen tienda son propietarios de sus tiendas, por lo que no incurren en gastos, en consecuencia, no es lógico que tengan menores utilidades; b) En +e no parece lógico que los mcs que tienen estudios superiores obtengan menos utilidad que los que no tienen formación. De los outliers, 4 de los 5 tienen estudios superiores; y en apreciación de los asesores, los mcs con mayor educación logran mayor utilidad. En las reuniones de discusión con los asesores de $+\mathbf{e}$ se ha decidido poner atención en el trabajo de campo y volver a analizar estas variables.

\section{Explicación de Ingresos por Venta Directa}

Se ha logrado desarrollar un modelo que explica el $67 \%$ de los Ingresos Brutos por Ventas de los mcs, con 4 variables lógicas, con las cuáles se puede hacer afirmaciones con un nivel de certeza mayor al 95\%: 1) Es lógico que los mcs que tienen habilidades buenas hagan más venta que los que tienen pocas habilidades, porque logran convencer más a los clientes y gestionan mejor su emprendimiento; 2) Si los mcs trabajan más días visitando comunidades $_{L}$ es lógico que logren más venta porque están accediendo a una clientela mayor $y_{i}$ 3) Lo mismo sucede cuando los mcs dedican más días de trabajo en ferias.

Respecto de las variables identificadas en la revisión bibliográfica hay que indicar que la variable Ingresos suficientes se incorpora como Ingreso Bruto Ventas e Ingreso Bruto Distribución. 
El aporte de la investigación, por comparación con la bibliografía revisada, es su abordaje cuantitativo, la discriminación por orden de importancia de las variables y la relación explicativa entre los ingresos por venta directa y sus predictores.

El modelo contiene 2 variables que no son lógicas: a) No es lógico afirmar que en los municipios en los que la población da una prioridad media a la energía, los mcs realicen menores ventas que en los municipios con prioridad baja, porque esto supondría una demanda menor. b) No es lógico indicar que mcs que gozan de una reputación aceptable, realizarán menos ventas que los que gozan de poca reputación, porque gozarían de menor confianza de los clientes.

\section{Explicación de Ingresos por Venta Distribución}

Se desarrolló un modelo que explica el 92\% de los Ingresos Brutos por Distribución de los mcs, con una variable lógica, con la cual se puede hacer afirmaciones con un nivel de certeza mayor al 95\%: Es lógico que en los municipios en los que las "autoridades municipales dan una prioridad alta a la energía", los mcs realicen más ventas por distribución. Se puede indicar que ésta es la variable crítica para lograr ventas por distribución; sin embargo, por constatación de campo se considera que para consolidar las ventas son necesarios robustos procesos de socialización que lleguen hasta los clientes.

\section{CONCLUSIONES}

El estudio que se presenta se basa en las investigaciones desarrolladas sobre la temática, reflexionándolas y adecuándolas al contexto de una $\mathrm{MC}$ en energías renovables en Bolivia, y responde a las urgencias del trabajo cotidiano de +e. Aporta un enfoque cuantitativo explicativo y de priorización. Inicialmente mide la rentabilidad los mcs de $+\mathbf{e}$ en términos de utilidad (ingresos totales - gastos totales), probando que ésta ha sido suficiente. Luego desarrolla un modelo regresional de la rentabilidad explicando que depende, en relación directa y por orden de importancia, de la "Prioridad que da la población a la energía", la "Competencia", la "Reputación de los mcs", los "Días que trabajan al mes", la "Edad que tienen", y la "Publicidad".

Finalmente, desarrolla otros dos modelos regresionales que prueban que las Ventas Directas de los mcs (variable intrínseca de MC) dependen, en relación directa y por orden de importancia, de sus "Habilidades como emprendedor", de los "Días de trabajo en las comunidades campesinas" y los "Días de trabajo en las ferias"; que las Ventas por Distribución (variable específica a +e) dependen de la "Prioridad que dan las autoridades municipales a la energía".

La MC +e, a futuro, deberá desarrollar investigaciones que aporten a la discusión de la temática: la rapidez en el logro de la rentabilidad, la continuidad o discontinuidad de la rentabilidad, las variables ilógicas resultantes de la investigación y la aplicación práctica de los hallazgos en el trabajo cotidiano de $+\mathbf{e}$.

\section{REFERENCIAS}

Beck, S; Deelder, W \& Miller, R. (2010). Franchising in Frontier Markets What's Working,What's Not, and Why. Dalberg Global Development Advisors. Innovations / Winter. p 163 - 162

Burand, D \& Koch, D. W. (2010). Microfranchising: A Business Approach to Fighting Poverty. Franchise Law Journal, 
Volume 30, Number 1, Summer 2010. (C) 2010 by the American Bar Association

Conde, A. (2013) MC: Desarrollo de un Modelo de Reproducción de Microfranquicias en México. Pequeñas y Medianas Empresas Franquiciantes (PYME). FUNDES. México

Fairbourne, J. S. (2007) "Why microfranchising is needed now: introduction and book Overview" in Microfranchising: creating wealth at the bottom of the pyramid / edited by Jason S. Fairbourne, Stephen W. Gibson and W. Gibb Dyer. Edward Elgar Publishing, Inc. Massachusetts. USA. págs 114

Hair, Jr. F; Black, W. C.; BABIN, J. B. \& Anderson R. E. (2014). Multivariate Data Analysis (7th Edition) 7th Edition. Pearson New International Edition. Edinburgh. England

Hurlimann, T. (2011). Micro-Franchising: Application and Success Factors. Kellogg School of Management.
http://api.ning.com/files/zkGb0X42pMTDrQ TwC5cbSBJ8DviNFc75zx1SrAIqJLDRYqbhve $\mathrm{KJ}$ olvuXyOMSGbGeMw6N0M5p4USC172OQoixpmJT 2COtaO/MicrofranchisingKellogManageme nt.pdf

Jacoby, W. G. (2017). Using standardized coefficients in a Regression equation with dummy variables. Michigan State University. Department of Political Science http://polisci.msu.edu/jacoby/msu/pls802/h andouts/dumvars/Dummy\%20Vars\%20and \%20Std\%20Coeffs,\%202017\%20Handout.pd $\mathrm{f}$

Lehr, D. (2008). Microfranchising at the Base of the Pyramid. Working paper

Sierraalta, Ni Terrazas, J. M. \& Huyhuati, M. (2017). Análisis Económico Financiero "Mas Energía" 2015 - octubre 2016. Energética. Cochabamba. Bolivia 